\title{
FREE VIBRATION ANALYSIS OF MULTIPLY CONNECTED PLATES USING THE METHOD OF FUNDAMENTAL SOLUTIONS
}

\author{
Ying-Te Lee ${ }^{1}$, Jeng-Tzong Chen ${ }^{1}$ and I-Lin Chen ${ }^{2}$ \\ ${ }^{1}$ Department of Harbor and River Engineering, National Taiwan Ocean University, \\ Keelung 20224, Taiwan \\ ${ }^{2}$ Department of Naval Architecture, National Kaohsiung Marine University, \\ Kaohsiung 81443, Taiwan
}

\begin{abstract}
In this paper, the method of fundamental solutions (MFS) for solving the eigenfrequencies of multiply connected plates is proposed. The coefficients of influence matrices are easily determined when the fundamental solution is known. True and spurious eigensolutions appear at the same time. It is found that the spurious eigensolution using the MFS depends on the location of the inner boundary where the fictitious sources are distributed. To verify this finding, mathematical analysis for the appearance of spurious eigenequations using degenerate kernels and circulants is done by demonstrating an annular plate with a discrete model. In order to obtain the true eigensolution, the Burton \& Miller method is utilized to filter out the spurious eigensolutions. One example is demonstrated analytically and numerically to see the validity of the present method.
\end{abstract}

Keywords: method of fundamental solutions, biharmonic equation; circulant; degenerate kernel; SVD updating document; Burton \& Miller method

\section{INTRODUCTION}

The method of fundamental solutions (MFS) is a numerical approach as well as finite difference method (FDM), finite element method (FEM) and boundary element method (BEM). This method was attributed to Kupradze in 1964 [1]. The MFS was applied to many problems (Refs.[2-4]), and can be regarded as one kind of meshless method. It has several advantages over boundary element 
method, e.g., no boundary integrals, no singularity and mesh-free model. Although MFS has been applied to solve many engineering problems, most of them are for cases of simply connected domains. Chen et al. have tried to solve the eigenproblem of multiply connected membrane and found that spurious eigenvalues also appear (Ref. [5]) as well as BEM (Ref. [6]). We may wonder whether spurious solutions also occur for the plate case rather than membrane.

In this paper, the MFS for solving the eigenfrequencies of annular plate is proposed. The occurring mechanism of the spurious eigensolution of an annular plate is studied analytically. The degenerate kernels and circulants are employed to determine the spurious eigensolution. In order to filter out the spurious eigenvalues, singular value decomposition updating technique and Burton \& Miller method are utilized. An annular case is demonstrated analytically to see the validity of the present method.

\section{ANALYTICAL DERIVATION OF FREE VIBRATION FOR ANNULAR PLATE USING THE METHOD OF FUNDAMENTAL SOLUTIONS}

The governing equation for an annular plate vibration in Figure 1 is the biharmonic equation as follows:

$$
\nabla^{4} u(x)=\lambda^{4} u(x), \quad x \in \Omega
$$

where $\nabla^{4}$ is the biharmonic operator, $u$ is the lateral displacement, $\lambda^{4}=$ $\omega^{2} \rho_{0} h / D, \lambda$ is the frequency parameter, $\omega$ is the angular frequency, $\rho_{0}$ is the surface density, $D$ is the flexural rigidity expressed as $D=E h^{3} / 12\left(1-v^{2}\right)$ in terms of Young's modulus $E$, the Poisson ratio $v$ and the plate thickness $h, \Omega$

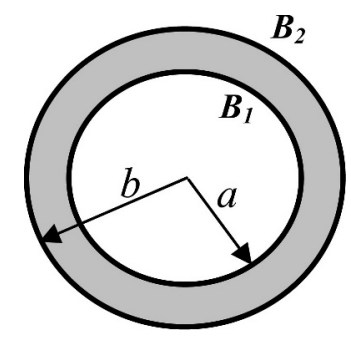

Figure 1. An annular problem. 


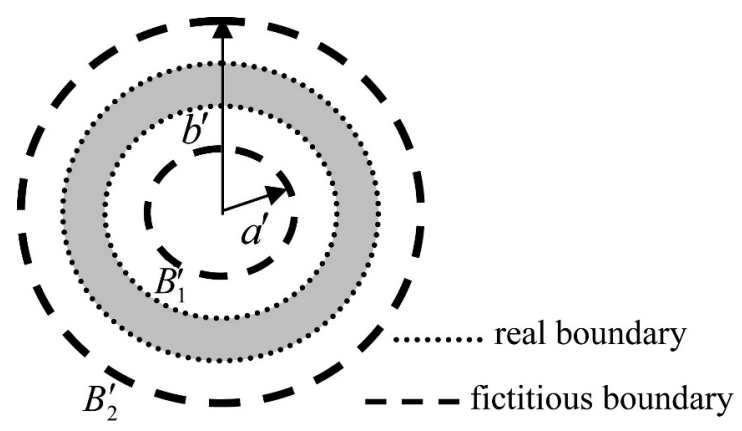

Figure 2. Figure sketch for source distribution.

is the domain of the thin plate. The fundamental solution is chosen as

$$
U(s, x)=\frac{1}{8 \lambda^{2}}\left[Y_{0}(\lambda r)-i J_{0}(\lambda r)+\frac{2}{\pi}\left(K_{0}(\lambda r)-i I_{0}(\lambda r)\right)\right]
$$

where $r \equiv|s-x|, i^{2}=-1, J_{0}(\lambda r)$ and $Y_{0}(\lambda r)$ are the first kind and second kind zeroth-order Bessel functions, respectively, $I_{0}(\lambda r)$ and $K_{0}(\lambda r)$ are the first and second kind zeroth-order modified Bessel functions, respectively. Based on the MFS, we can represent the displacement field of plate vibration by

$$
u\left(x_{i}\right)=\sum_{j=1}^{2 N} P\left(s_{j}, x_{i}\right) \phi_{j}+\sum_{j=1}^{2 N} Q\left(s_{j}, x_{i}\right) \varphi_{j},
$$

where $2 N$ is the number of fictitious source nodes. $\phi_{j}$ and $\varphi_{j}$ are the known densities with respect to $P$ and $Q$. The two kernels ( $P$ and $Q$ ) are obtained from either the two of the kernel $U(s, x)$ and the other three kernels, $\Theta(s, x), M(s, x)$ and $V(s, x)$ (Ref. [7]). The slope $(\theta)$, normal moment $(m)$ and effective shear force $(v)$, are also obtained as reference (Ref. [7]). In order to derive the exact eigensolution, degenerate kernel and circulant are considered for an annular plate. The field and source points are distributed as shown in Figure 2. Here, we consider the clamped case $(u=0$ and $\theta=0)$ by using $U$ and $\Theta$ kernels. We distributed $2 N$ field points on the real boundary, and the same $2 N$ sources are distributed on the fictitious boundary. By matching the boundary condition, we obtain

$$
\left[S M^{c c}\right]\left\{\begin{array}{c}
\phi \\
\phi 2 \\
\varphi 1 \\
\varphi 2
\end{array}\right\}=\left[\begin{array}{cccc}
U 11 & U 12 & \Theta 11 & \Theta 12 \\
U 21 & U 22 & \Theta 21 & \Theta 22 \\
U 11_{\theta} & U 12_{\theta} & \Theta 11_{\theta} & \Theta 12_{\theta} \\
U 21_{\theta} & U 22_{\theta} & \Theta 21_{\theta} & \Theta 22_{\theta}
\end{array}\right]_{8 N \times 8 N} \quad\left\{\begin{array}{c}
\phi 1 \\
\phi 2 \\
\varphi 1 \\
\varphi 2
\end{array}\right\}=\{0\}
$$


where $\{\phi 1\},\{\phi 2\},\{\varphi 1\}$ and $\{\varphi 2\}$ are the generalized coefficients for $B_{1}$ and

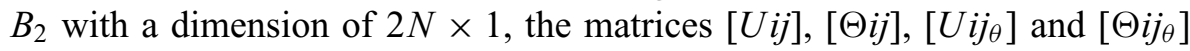
mean the influence matrices of $U, \Theta, U_{\theta}$ and $\Theta_{\theta}$ kernels which are obtained by collocating the field and source points on $B_{i}$ and $B_{j}^{\prime}$ with a dimension of $2 N \times 2 N$, respectively. For the existence of nontrivial solution, the determinant of the matrix vs. the eigenvlaue must be zero, i.e.,

$$
\operatorname{det}\left[S M^{c c}\right]=\prod_{m=-(N-1)}^{N} \operatorname{det}\left(\left[T_{m}^{c c}\right]\left[S_{m}^{U \Theta}\right]\right)=0,
$$

where

$$
\left[T_{m}^{c c}\right]=\left[\begin{array}{cccc}
J_{m}(\lambda a) & Y_{m}(\lambda a) & I_{m}(\lambda a) & K_{m}(\lambda a) \\
J_{m}(\lambda b) & Y_{m}(\lambda b) & I_{m}(\lambda b) & K_{m}(\lambda b) \\
J_{m}^{\prime}(\lambda a) & Y_{m}^{\prime}(\lambda a) & I_{m}^{\prime}(\lambda a) & K_{m}^{\prime}(\lambda a) \\
J_{m}^{\prime}(\lambda b) & Y_{m}^{\prime}(\lambda b) & I_{m}^{\prime}(\lambda b) & K_{m}^{\prime}(\lambda b)
\end{array}\right]
$$

and

$$
\begin{aligned}
& {\left[S_{m}^{U \Theta}\right]=} \\
& {\left[\begin{array}{cccc}
-i J_{m}\left(\lambda a^{\prime}\right) & Y_{m}\left(\lambda b^{\prime}\right)-i J_{m}\left(\lambda b^{\prime}\right) & -i J_{m}^{\prime}\left(\lambda a^{\prime}\right) & Y_{m}^{\prime}\left(\lambda b^{\prime}\right)-i J_{m}^{\prime}\left(\lambda b^{\prime}\right) \\
J_{m}\left(\lambda a^{\prime}\right) & 0 & J_{m}^{\prime}\left(\lambda a^{\prime}\right) & 0 \\
-(-1)^{m} i \frac{2}{\pi} I_{m}\left(\lambda a^{\prime}\right) & \frac{2}{\pi}\left[k_{m}\left(\lambda b^{\prime}\right)-(-1)^{m} i I_{m}\left(\lambda b^{\prime}\right)\right] & -(-1)^{m} i \frac{2}{\pi} I_{m}^{\prime}\left(\lambda a^{\prime}\right) & \frac{2}{\pi}\left[k_{m}^{\prime}\left(\lambda b^{\prime}\right)-(-1)^{m} i I_{m}^{\prime}\left(\lambda b^{\prime}\right)\right] \\
\frac{2}{\pi} I_{m}\left(\lambda a^{\prime}\right) & 0 & \frac{2}{\pi} I_{m}^{\prime}\left(\lambda a^{\prime}\right) & 0
\end{array}\right] .}
\end{aligned}
$$

It is noted that the matrix $\left[T_{m}^{c c}\right]$ denotes the matrix of true eigenequation for the $C-C$ case and the matrix $\left[S_{m}^{U \Theta}\right]$ denotes the matrix of spurious eignequation in the $U-\Theta$ formulation after comparing with the analytical solution for the annular plate (Ref. [8]). The matrix in Equation (7) can be further decomposed into

$$
\begin{aligned}
\operatorname{det}\left[S_{m}^{U \Theta}\right]= & \left|\begin{array}{cc}
J_{m}\left(\lambda a^{\prime}\right) & J_{m}^{\prime}\left(\lambda a^{\prime}\right) \\
I_{m}\left(\lambda a^{\prime}\right) & I_{m}^{\prime}\left(\lambda a^{\prime}\right)
\end{array}\right| \\
& \times\left|\begin{array}{cc}
Y_{m}\left(\lambda b^{\prime}\right)-i J_{m}\left(\lambda b^{\prime}\right) & Y_{m}^{\prime}\left(\lambda b^{\prime}\right)-i J_{m}^{\prime}\left(\lambda b^{\prime}\right) \\
K_{m}\left(\lambda b^{\prime}\right)-i(-1)^{m} I_{m}\left(\lambda b^{\prime}\right) & K_{m}^{\prime}\left(\lambda b^{\prime}\right)-i(-1)^{m} I_{m}^{\prime}\left(\lambda b^{\prime}\right)
\end{array}\right|=0
\end{aligned}
$$

Since the latter part of Equation (8) is never zero, the spurious eigenequation depends on $a^{\prime}$. It is noted that the spurious eigensolution happens to be true 


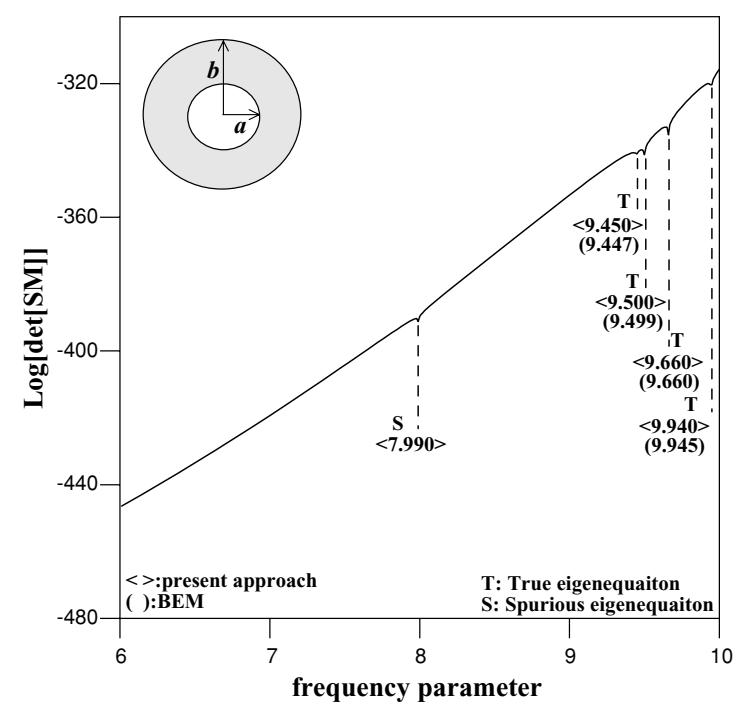

Figure 3. The determinant vs. frequency parameter by using the $U-\Theta$ formulation.

eigensolution of the clamped circular plate with a radius $a^{\prime}$. Therefore, the positions of spurious eigenvalues for the annular problem depend on the location of inner fictitious boundary $a^{\prime}$ where the sources are distributed.

\section{A NUMERICAL EXAMPLE}

An annular plate with the inner radius of 0.5 meter and the outer radius of 1 meter are considered, respectively. The source points are distributed at $a^{\prime}=0.4$ meter and $b^{\prime}=1.2$ meter. Forty-six nodes are uniformly distributed on the inner and outer fictitious boundaries. Figure 3 shows the determinant vs. frequency parameter by using the $U-\Theta$ formulation. The drop location indicates the possible eigenvalues. Figure 4 shows the determinant vs. frequency parameter by using the Burton \& Miller method for the annular plate. It is found that the appearance of spurious eigenvalues is suppressed. After comparing the result with the analytical solution, good agreement is made.

\section{CONCLUSIONS}

The mathematical analysis has shown that spurious eigenvalues occur by using degenerate kernels and circulants when the method of fundamental solutions is used to solve the eigenvalue of annular plates. The positions of 


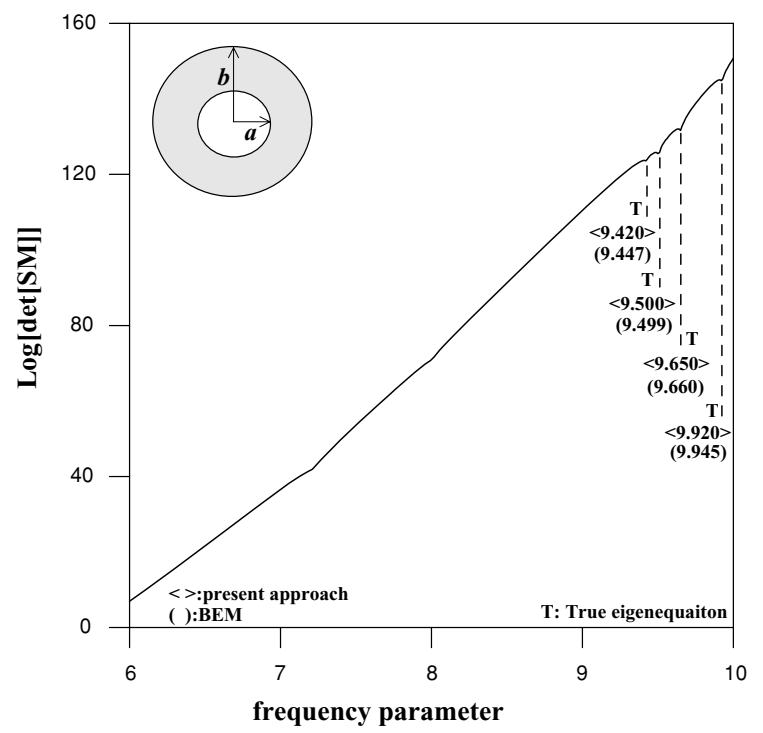

Figure 4. The determinant vs. frequency parameter by using the $U-\Theta$ formulation in conjunction with Burton \& Miller method.

spurious eigenvalues for the annular problem depend on the location of inner fictitious boundary where the sources are distributed. The spurious eigenvalues in the annular problem are found to be the true eigenvalues of the associated simply connected problem bounded by the inner sources. We have employed the Burton \& Miller method to filter out the spurious eigenvalues successfully.

\section{REFERENCES}

1. V.D. Kupradze (1964), A method for the approximate solution of limiting problems in mathematical physics. Computational Mathematics and Mathematical Physics, 4, pp. 199 205.

2. G. Fairweather and A. Karageorghis (1998), The method of fundamental solutions for elliptic boundary value problems. Advances in Computational Mathematics, 9, pp. 69-95.

3. C.S. Chen, M.A. Golberg and Y.C. Hon (1998), The method of fundamental solutions and quasi-Monte-Carlo method for diffusion equations. International Journal for Numerical Methods in Engineering, 43, pp. 1421-35.

4. A. Karageorghis (2001), The method of fundamental solutions for the calculation of the eigenvalues of the Helmholtz equation. Applied Mathematics Letters, 14, pp. $837-42$. 
5. J.T. Chen, S.Y. Lin, K.H. Chen and I.L. Chen (2004), Mathematical analysis and numerical study of true and spurious eigenequations for free vibration of plates using real-part BEM. Computational Mechanics, 34, pp. 165-180.

6. J.T. Chen, L.W. Liu and H.-K. Hong (2003), Spurious and true eigensolutions of Helmholtz BIEs and BEMs for a multiply-connected problem. In Proceedings of the Royal Society London Series A, 459, pp.1891-925.

7. J.T. Chen, I.L. Chen and Y.T. Lee (2004), Eigensolutions of multiply-connected membranes using the method of fundamental solutions. Engineering Analysis with Boundary Elements, Revised.

8. W. Leissa (1969), Vibration of Plates. NASA SP-160. 